\title{
Complex sarcolemmal invaginations mimicking myotendinous junctions in a case of Laing early-onset distal myopathy
}

Gerald F. Reis ${ }^{1}$, Grant de la Motte ${ }^{2}$, Rebecca Gooding ${ }^{3}$, Nigel G. Laing ${ }^{4}$, and Marta Margeta ${ }^{1 *}$

${ }^{1}$ Department of Pathology, University of California, San Francisco, CA, US

2 Palo Alto Medical Foundation, Santa Cruz, CA, US

${ }^{3}$ Neurogenetics Unit, PathWest Laboratory Medicine, QEII Medical Centre, Nedlands, AU

${ }^{4}$ Centre for Medical Research, University of Western Australia and Harry Perkins Institute of Medical Research, QEII Medical Centre, Nedlands, AU

* Corresponding author: UCSF Pathology, Box 0511

513 Parnassus Ave., HSW-514

San Francisco, CA 94143

Phone: 415-514-0228

Fax: 415-514-3165

E-mail: Marta.Margeta@ucsf.edu

Running Title: Type 1 fiber clefting in MPD1 muscle

This article has been accepted for publication and undergone full peer review but has not been through the copyediting, typesetting, pagination and proofreading process which may lead to differences between this version and the Version of Record. 


\section{Abstract}

Distal myopathies are a group of clinically and pathologically overlapping muscle diseases that are genetically complex and can represent a diagnostic challenge. Laing early-onset distal myopathy (MPD1) is a form of distal myopathy caused by mutations in the MYH7 gene, which encodes the beta myosin heavy chain protein expressed in type 1 skeletal muscle fibers and cardiac myocytes. Here, we present a case of genetically-confirmed MPD1 with a typical clinical presentation but distinctive light microscopic and ultrastructural findings on muscle biopsy. A 39-year-old professional male cellist presented with a bilateral foot drop that developed by age 8; analysis of the family pedigree showed an autosomal dominant pattern of inheritance. The physical exam demonstrated bilateral weakness of ankle dorsiflexors, toe extensors, and finger extensors; creatine kinase level was normal. Biopsy of the quadriceps femoris muscle showed predominance and hypotrophy of type 1 fibers; hybrid fibers with co-expression of slow and fast myosin proteins, both in highly atrophic and normal size range; moth-eaten fibers and minicores; lack of rimmed vacuoles; and rare desmin-positive eosinophilic sarcoplasmic inclusions. In addition to these abnormalities often observed in MPD1, the biopsy demonstrated frequent clefted fibers with complex sarcolemmal invaginations; on ultrastructural examination, these structures closely mimicked myotendinous junctions but were present away from the tendon and were almost exclusively found in type 1 fibers. Sequencing analysis of the MYH7 gene in the index patient and other affected family members demonstrated a previously described heterozygous c.4522_4524delGAG (p.Glu1508del) mutation. This case widens the pathologic spectrum of MPD1 and highlights the pathologic and clinical variability that can accompany the same genetic mutation, suggesting a significant role for modifier genes in MPD1 pathogenesis.

\section{Keywords:}

distal myopathy; slow myosin; type 1 fibers; clefted fibers; myotendinous junction-like structures 


\section{Introduction}

Distal myopathies are a group of muscle disorders that share a distinctive clinical presentation preferential weakness of distal skeletal muscles - but are otherwise heterogeneous: more than 20 genetically distinct forms have been described, some presenting early in life and others in adulthood $^{1}$. Laing early-onset distal myopathy (MPD1) is a form of autosomal dominant distal myopathy that is caused by mutations in the MYH7 gene; $M Y H 7$ encodes the beta myosin heavy chain protein, which is expressed in type 1 skeletal muscle fibers and cardiac myocytes ${ }^{2}$. (Two other clinical phenotypes - hypertrophic/dilated cardiomyopathy ${ }^{3}$ and myosin storage/hyaline body myopathy ${ }^{4}$ - can be caused by MYH7 mutations; it is thought that the disease phenotype mostly depends on the location of mutation within the myosin protein, with MPD1 mutations primarily affecting the myosin tail that is critical for coiled-coil formation and binding of the myosin-associated proteins ${ }^{2}$.) In the large majority of cases, MPD1 presents with early-onset ankle dorsiflexion weakness. Symptoms first appear in early childhood and may also include weakness of neck flexors and a hanging big toe; in some cases, weakness of shoulder muscles and finger extensors can occur, as well as axial weakness that can lead to scoliosis ${ }^{2,5,6}$. The disease typically progresses very slowly and the majority of patients retain ambulatory function throughout life; creatine kinase (CK) level is usually not elevated. The muscle biopsy findings are generally nonspecific, with type 1 fiber atrophy and predominance observed in the majority of described cases ${ }^{6,7}$; however, muscle morphology can vary significantly even among the members of the same family ${ }^{8,9}$. Here, we present a case of genetically-confirmed MPD1 with a typical clinical presentation but distinctive light microscopic and ultrastructural findings on muscle biopsy.

\section{Clinical Summary}

A left-handed professional male cellist with a history of bilateral foot drop form early childhood presented for evaluation and genetic counseling at the age of 39. He had lost the ability to dorsiflex both feet at the age of 4-5 and had developed complete bilateral foot drop over the ensuing 2-3 years, at which point the disease appeared to stabilize with no further progression noticed subjectively. The initial diagnostic work-up, including electromyography, was performed at the age of 9 and yielded no specific diagnosis. At 39, the patient reported no 
difficulty ambulating and was able to ride a bicycle and surf regularly. The physical exam showed normal muscle bulk and tone; however, bilateral weakness of ankle dorsiflexors (1/5), toe extensors (1/5), and finger extensors (4+/5) was noted. Neck flexors were mildly weak $(4+/ 5)$, while neck extensors and axial muscles showed normal strength. On electromyography, myopathic features with minimal muscle membrane instability were detectable in both proximal and distal muscle groups, with greater involvement of distal muscles; among proximal muscle groups, the biceps brachii muscles were more involved than the quadriceps femoris muscles. Nerve conduction studies were unremarkable. Laboratory tests included a normal CK level of 222 U/L. Echocardiography was essentially normal, with only mildly reduced left ventrícular systolic wall motion.

Based on the family history provided by the proband and other family members, the disease first manifested in the proband's paternal grandmother, showing an autosomal dominant pattern of inheritance in subsequent generations (Fig. 1). The proband's family resides in a different state and was not available for physical examination, but he reported that affected family members showed a stereotypical pattern of involvement, with bilateral foot drop that appears in early childhood (5-8 years of age on average) and is followed by a non-progressive or minimally progressive disease; per family's report, exercise appears to be protective, with the degree of physical activity showing inverse relation to the age of symptom onset in the affected individuals. According to the information provided by the proband's family members, the proband's father (II/5), two uncles (II/4 and II/8), and younger brother (III/4) all manifest complete bilateral foot drop. In the fourth generation, the proband's nephew IV-1 was asymptomatic at age 16, while the proband's nieces IV/2 (age 14) and IV/3 (age 11) showed mild motor abnormalities suspicious for MPD1 (IV/2 developed mild left foot drop by age 10, while IV/3 could not walk on heels but was otherwise able to walk normally at the time of genetic testing); the fourth generation siblings have not been evaluated by a neurologist. Per report, some of the affected individuals also developed cardiac disease ranging from a mild decrease in the cardiac ejection fraction (II/5 and III/4) to a heart failure requiring pacemaker at age 60 (II/4); however, detailed medical information was not available. 


\section{Pathological and Genetic Findings}

The patient underwent muscle biopsy from the left quadriceps femoris; the biopsy was taken from the central portion of the vastus lateralis, at a site approximately equidistant from both tendons. The flash-frozen muscle tissue was evaluated using a standard panel of muscle stains, (hematoxylin and eosin [H\&E]; modified trichrome; ATPase, $\mathrm{pH}=$ 9.4; NADH-TR; SDH; and COX). Immunoperoxidase stains for desmin (mouse monoclonal antibody, clone D33; Cell Marque, 1:5 dilution), slow myosin (mouse monoclonal antibody, clone WB-MHCff; Leica Biosystems, 1:50 dilution), and fast myosin (mouse monoclonal antibody, clone WB-MHCf; Leica Biosystems, 1:20 dilution) were also performed on the frozen material. H\&E-stained cryosections (Fig. 2A) showed moderate variation in muscle fiber diameters (10-100 $\mu \mathrm{M})$, scattered subsarcolemmal nuclear aggregates, and mild endomysial fibrosis without degenerating or regenerating fibers; notably, a number of randomly distributed fibers of variable size appeared clefted or fragmented, showing complex sarcolemmal invaginations ( 60 clefted fibers on a 15 $\mathrm{mm}^{2}$ cross-section). ATPase stain (Fig. $2 \mathrm{~B}$ ) showed type 1 fiber predominance ( 6:1 type $1 /$ type 2 fiber ratio); while not atrophic overall, type 1 fibers were smaller than type 2 fibers on average (type 1 fiber diameter: $53.4 \pm 19.9 \mu \mathrm{m}$ [mean \pm S.D.], type 2 fiber diameter: $93.5 \pm 25.3$ $\mu \mathrm{m} ; \mathrm{p}<0.0001$, t-test, $\mathrm{n}=31-39$ ). Interestingly, sarcolemmal invaginations were seen in 55 type 1 fibers but only 2 type 2 fibers ( 28:1 clefted type 1 / clefted type 2 fiber ratio), suggesting preferential involvement of type 1 fibers. NADH-TR histochemistry showed a spectrum of sarcoplasmic abnormalities that ranged from a moderate degree of "moth-eateness" to welldeveloped mini-cores (Fig. 2C). Trichrome stain showed rare eosinophilic cytoplasmic inclusions but no rimmed vacuoles (Fig. 2D). Desmin immunoperoxidase stain demonstrated rare desmin-positive subsarcolemmal inclusions (Fig. 2E), likely corresponding to eosinophilic inclusions seen on trichrome stain. Clefted fibers with membrane invaginations were easily identifiable on Toluidine blue-stained resin sections (Fig. 2F). Aberrant fast myosin expression was seen in scattered type 1 fibers, some of which were highly atrophic while others had normal diameter (Fig. 3); sarcoplasmic inclusions were myosin-negative.

Ultrastructural analysis demonstrated focal areas of myofibrillar disorganization with granulofilamentous material and Z-band streaming (Fig. 4A), likely corresponding to sarcoplasmic inclusions seen on trichrome and desmin stains. The most striking ultrastructural 
feature was the presence of abnormal membrane invaginations reminiscent of myotendinous junctions (MTJs) (Figs. 4B-C). The myofibrillar apparatus abutting sarcolemmal invaginations was disorganized and contained mitochondria with dense matrical granules and paracrystalline arrays (Fig. 4D); in contrast, the mitochondria away from these areas were morphologically unremarkable.

The clinical presentation and general muscle biopsy features suggested MPD1 as the most likely diagnosis; we thus performed sequencing analysis of the $\mathrm{MYH7}$ gene as described previously $^{6}$. The sequencing revealed heterozygous deletion of a GAG triplet at position $4522-$ 4524 (Fig. 5); this mutation is predicted to result in the loss of a glutamate residue at position 1508 of the beta myosin heavy chain protein [c.4522_4524delGAG (p.Glu1508del)] and has already been linked to MPD1 in several unrelated families throughout the world ${ }^{6,10,11}$. The same heterozygous mutation was present in the affected family members III-4, IV-2 and IV-3, while the unaffected family member IV-1 showed a normal MYH7 genotype.

\section{Discussion}

At the time of its phenotypic and genetic characterization ${ }^{2,5}$, MPD1 was thought to have a relatively stereotypic clinical presentation and disease course; in contrast, the underlying muscle pathology showed considerable heterogeneity ${ }^{7}$. Since then, both the clinical and pathologic spectra of MPD1 have significantly widened, with diverse muscle group involvement and varying pathology seen even in cases with the same underlying mutation ${ }^{8,9}$. In the current report, we describe a patient with the clinical presentation and disease course typical for classic MPD1, but with unusual light microscopic and ultrastructural features on muscle biopsy (Figs. 2 and 4).

Muscle biopsies from MPD1 patients usually show type 1 fiber predominance, fiber size variation with type 1 fiber atrophy, hybrid fibers that express both slow and fast myosin proteins, and a spectrum of sarcoplasmic abnormalities that range from moth eaten fibers to welldeveloped mini-cores ${ }^{6-9}$; rimmed vacuoles, which are frequently seen in other distal myopathies, are usually not present ${ }^{1,7}$. Less commonly, the pathologic spectrum of MPD1 includes eosinophilic inclusion bodies ${ }^{12}$ and mitochondrial abnormalities ${ }^{9,13}$. In the current case, the biopsy of a clinically less involved quadriceps muscle showed most of these features, including type 1 fiber predominance and fiber size variation (Fig. 2B); scattered hybrid fibers (including 
highly atrophic hybrid fibers thought to be pathognomonic for MPD16 ${ }^{6}$; Fig. 3); mini-cores (Fig. 2C) and rare desmin-positive eosinophilic sarcoplasmic inclusions (Figs. 2D-E); and disorganization of the myofibrillary apparatus / granulofilamentous material (Fig. 4A). Interestingly, while type 1 fibers were smaller than type 2 fibers on average, they were not actually atrophic (average diameter, $53.4 \mu \mathrm{m}$ ); this may be a function of the muscle chosen for biopsy (quadriceps femoris instead of tibialis anterior), but could also reflect the fact that the proband engages in a level of physical activity that is unusually high for an MPD1 patient. In addition to these commonly observed pathologic abnormalities, the current biopsy showed frequent fibers with extensive membrane invaginations (Figs. 2A, 2B, 2F) that on ultrastructural examination had an MTJ-like appearance (Figs. 4B-D).

MTJs are specialized structures that allow transmission of mechanical force from muscle fiber myofilaments to tendon collagen fibers ${ }^{14}$. On longitudinal view, MTJ consists of numerous muscle fiber projections that are limited by sarcolemma and basement membrane; these projections interdigitate with tendon processes composed of fibroblasts, fibroblast processes, and collagen fibers $^{15}$. On cross-sectional view of MTJ, muscle fibers appear fissured, with collagen fibers and fibroblast processes inside the clefts; sarcolemma abutting the clefts is thickened and overlies a sarcoplasmic zone with a Z-band-like electron density ${ }^{15,16}$. Clefted fibers observed in the proband's biopsy showed many classic MTJ features, including fibroblast processes and collagen fibrils within clefts and Z-band-like electron dense material under the sarcolemma (Figs. 4B-D). However, these MTJ-like structures were preferentially found in type 1 fibers (Fig. 2B) and were randomly distributed throughout the biopsy, a pattern that would not be expected for true MTJs. Furthermore, the biopsy was taken from the central part of the vastus lateralis, away from both tendons. Finally, mitochondria adjacent to the MTJ-like sarcolemmal invaginations in the proband's biopsy showed ultrastructural abnormalities (Fig. 4D) that have not been reported in association with true $\mathrm{MTJ}^{17,18}$. Taken together, we believe that fiber clefting/fragmentation accompanied by aberrant MTJ-like structure formation in type 1 fibers is a part of the MPD1 phenotype in this patient, widening the pathologic spectrum previously associated with this disease.

Sequencing of the MYH7 gene demonstrated the presence of a heterozygous c.4522_4524delGAG (p.Glu1508del) mutation in the proband and three other affected members of his family; this mutation was previously linked to MPD1 in (i) a French family, a Norwegian 
family, and a single Finnish patient ${ }^{10}$, (ii) a Belgian family ${ }^{11}$, and (iii) another US family . $^{6}$ Interestingly, muscle biopsies from other patients with p.Glu1508del mutation showed no unusual morphologic features and were not described as harboring MTJ-like structures; a similar lack of correlation between genetics and muscle pathology has been observed with two other MPD1 mutations (p.K1729del ${ }^{9}$ and p.Leu1597 Arg ${ }^{8}$ ). Interestingly, the p.Glu1508del patient cohort described to date also shows a significant clinical variability: while all patients presented with classic bilateral foot drop at an early age, the involvement of other muscle groups and the speed of disease progression were highly variable. In particular, the disease manifestations in the Belgian family ${ }^{11}$ appear to be quite severe, while the family described in the current report manifests a very mild clinical course (for example, the proband [currently age 41] is actively involved in multiple sports and continues to play cello professionally despite the development of mild finger extensor weakness; his father (II/5), a school orchestra conductor for 40 years, continues to regularly walk, bike, and lift weights at the age of 66). These discrepancies between genotype and phenotype suggest a significant role for the genetic background / modifier genes in MPD1 pathogenesis, a feature of the disease that will need to be explored in the future studies.

\section{Acknowledgements}

We are grateful to Dr. Timothy Rietz (Mayo Clinic) for coordinating blood sample collection from the proband's relatives and to the proband's mother, father and uncle for providing family history details. We would also like to thank Dr. Xianhong Wang for help with genomic DNA preparation and Ms. Christine Lin for assistance with figure preparation. This work was supported by Australian National Health and Medical Research Council Fellowship APP1002147. The authors declare no competing interests. Written informed consent for genetic testing was obtained for all tested individuals; in addition, written informed consent was obtained from the index patient for publication of this case report and any accompanying images.

\section{Authors' contributions}

MM and NL designed research; GM performed clinical evaluation of the proband; GR and MM performed pathologic evaluation; MM performed fiber quantification and statistical analysis; RG performed and NL interpreted molecular genetic analyses; MM acquired images 
and designed figures; MM and GR wrote the paper with a significant contribution by NL; MM communicated with the family, coordinated the genetic testing, and supervised the project. All authors read and approved the final manuscript.

\section{References}

1 Udd B. Distal myopathies--new genetic entities expand diagnostic challenge. Neuromuscul Disord. 2012; 22: 5-12.

2 Meredith C, Herrmann R, Parry C, Liyanage K, Dye DE, Durling HJ, et al. Mutations in the slow skeletal muscle fiber myosin heavy chain gene (MYH7) cause laing early-onset distal myopathy (MPD1). Am J Hum Genet. 2004; 75: 703-8.

3 Walsh R, Rutland C, Thomas R, Loughna S. Cardiomyopathy: a systematic review of diseasecausing mutations in myosin heavy chain 7 and their phenotypic manifestations. Cardiology. 2010; 115: 49-60.

4 Tajsharghi H, Thornell LE, Lindberg C, Lindvall B, Henriksson KG, Oldfors A. Myosin storage myopathy associated with a heterozygous missense mutation in MYH7. Ann Neurol. 2003; 54: 494-500.

5 Laing NG, Laing BA, Meredith C, Wilton SD, Robbins P, Honeyman K, et al. Autosomal dominant distal myopathy: linkage to chromosome 14. Am J Hum Genet. 1995; 56: 422-7.

6 Lamont PJ, Wallefeld W, Hilton-Jones D, Udd B, Argov Z, Barboi AC, et al. Novel mutations widen the phenotypic spectrum of slow skeletal/beta-cardiac myosin (MYH7) distal myopathy. Hum Mutat. 2014; 35: 868-79.

7 Lamont PJ, Udd B, Mastaglia FL, de Visser M, Hedera P, Voit T, et al. Laing early onset distal myopathy: slow myosin defect with variable abnormalities on muscle biopsy. J Neurol Neurosurg Psychiatry. 2006; 77: 208-15.

8 Clarke NF, Amburgey K, Teener J, Camelo-Piragua S, Kesari A, Punetha J, et al. A novel mutation expands the genetic and clinical spectrum of MYH7-related myopathies. Neuromuscul Disord. 2013; 23: 432-6.

9 Muelas N, Hackman P, Luque H, Garces-Sanchez M, Azorin I, Suominen T, et al. MYH7 gene tail mutation causing myopathic profiles beyond Laing distal myopathy. Neurology. 2010; 75: 732-41.

10 Dubourg O, Maisonobe T, Behin A, Suominen T, Raheem O, Penttila S, et al. A novel MYH7 mutation occurring independently in French and Norwegian Laing distal myopathy families and de novo in one Finnish patient. J Neurol. 2011; 258: 1157-63.

11 Van den Bergh PY, Martin JJ, Lecouvet F, Udd B, Schmedding E. Laing early-onset distal myopathy in a Belgian family. Acta Neurol Belg. 2014; 114: 253-6. 
12 Tasca G, Ricci E, Penttila S, Monforte M, Giglio V, Ottaviani P, et al. New phenotype and pathology features in MYH7-related distal myopathy. Neuromuscul Disord. 2012; 22: 640-7.

13 Finsterer J, Brandau O, Stollberger C, Wallefeld W, Laing NG, Laccone F. Distal myosin heavy chain-7 myopathy due to the novel transition c.5566G>A (p.E1856K) with high interfamilial cardiac variability and putative anticipation. Neuromuscul Disord. 2014; 24: 721-5.

14 Charvet B, Ruggiero F, Le Guellec D. The development of the myotendinous junction. A review. Muscles Ligaments Tendons J. 2012; 2: 53-63.

15 Mair WG, Tome FM. The ultrastructure of the adult and developing human myotendinous junction. Acta Neuropathol. 1972; 21: 239-52.

16 Ishikawa H. The Fine Structure of Myo-Tendon Junction in Some Mammalian Skeletal Muscles. Arch Histol Jpn. 1965; 25: 275-96.

17 Knudsen AB, Larsen M, Mackey AL, Hjort M, Hansen KK, Qvortrup K, et al. The human myotendinous junction: An ultrastructural and 3D analysis study. Scand J Med Sci Sports. 2014.

18 Ovalle WK. The human muscle-tendon junction. A morphological study during normal growth and at maturity. Anat Embryol (Berl). 1987; 176: 281-94. 


\section{Figure legends}

Figure 1. Family pedigree showing an autosomal dominant inheritance pattern. The proband (III/3) is indicated by the arrow; the disease was first noted in his paternal grandmother $(\mathrm{I} / 1)$.

Figure 2. Muscle biopsy findings: standard light microscopy. Muscle biopsy showed moderate variation in fiber diameters, mild endomysial fibrosis, and no degenerating/regenerating fibers. On H\&E-stained cryosections (A), randomly distributed muscle fibers showed clefting/fragmentation with extensive invaginations of sarcolemma (solid arrowheads). On ATPase stain (B; pH=9.4), membrane invaginations were almost exclusively confined to type 1 fibers, which comprised 85\% of the biopsy and were smaller in diameter than type 2 fibers. NADH-TR stain (C) showed a spectrum of abnormalities including moth eaten fibers and mini-cores (empty arrowheads). Trichrome stain (D) showed no rimmed vacuoles and only rare eosinophilic cytoplasmic inclusions (white arrow); on desmin immunoperoxidase stain (E), rare desmin-positive subsarcolemmal inclusions were present (black arrow). A clefted fiber is easily observed on Toluidine blue-stained resin section (F, solid arrowhead). Scale bars, A and F, $25 \mu \mathrm{m}$; B-E, $50 \mu \mathrm{m}$.

Figure 3. Muscle biopsy findings: myosin immunohistochemistry. (A) Slow myosin (brown staining) is expressed in type 1 fibers, which comprise most of the biopsy; the two type 2 fibers in this field (asterisks) show complete absence of staining. (B) In addition to type 2 fibers (asterisks), fast myosin is expressed in a subset of hybrid fibers that also express slow myosin. While some hybrid fibers (solid arrowheads) were in the mildly atrophic to normal size range, others (open arrowheads) were very small and barely visible on the slow myosin stain. Scale bar, $50 \mu \mathrm{m}$.

Figure 4. Muscle biopsy findings: electron microscopy. (A) Electron microscopy showed focal areas of myofibrillar disorganization with granulofilamentous material and Z-band streaming. (B-D) Multiple fibers showed MTJ-like sarcoplasmic clefting, with electron dense material under the sarcolemma; collagen fibrils (“CF”) and fibroblasts / fibroblast processes (arrows) were present within the clefts. The myofibrillar apparatus abutting the membrane invaginations was focally disorganized and contained mitochondria with dense matrical granules 
(electron-dense deposits in the mitochondrial matrix; open arrowheads) and paracrystalline arrays (solid arrowheads). Scale bars: A and C-D, $0.5 \mu \mathrm{m}$; B, $2 \mu \mathrm{m}$.

Figure 5. MYH7 gene sequencing chromatogram. Sequencing chromatogram from the patient's MYH7 gene (bottom) shows heterozygous deletion of GAG triplet at position 45224524, which results in a deletion of a glutamate residue at position 1508 of the beta myosin heavy chain protein [c.4522_4524delGAG (p.Glu1508del)]. 
Figure 1

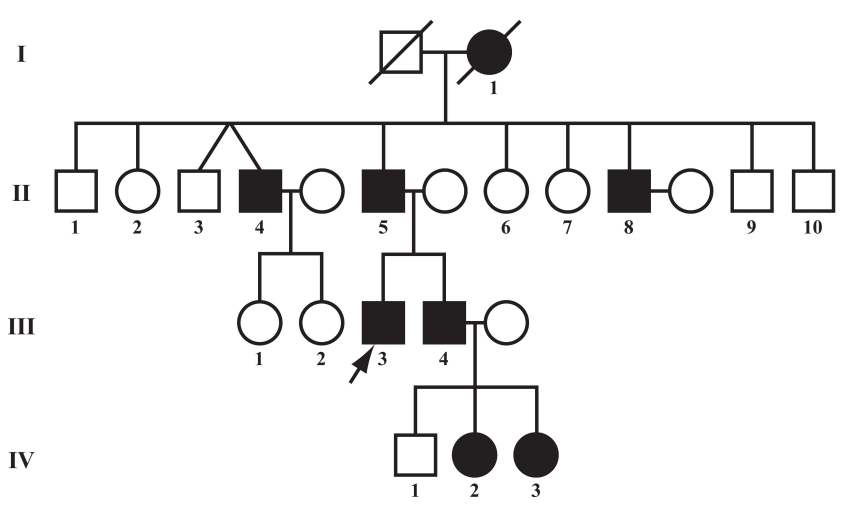


Figure 2
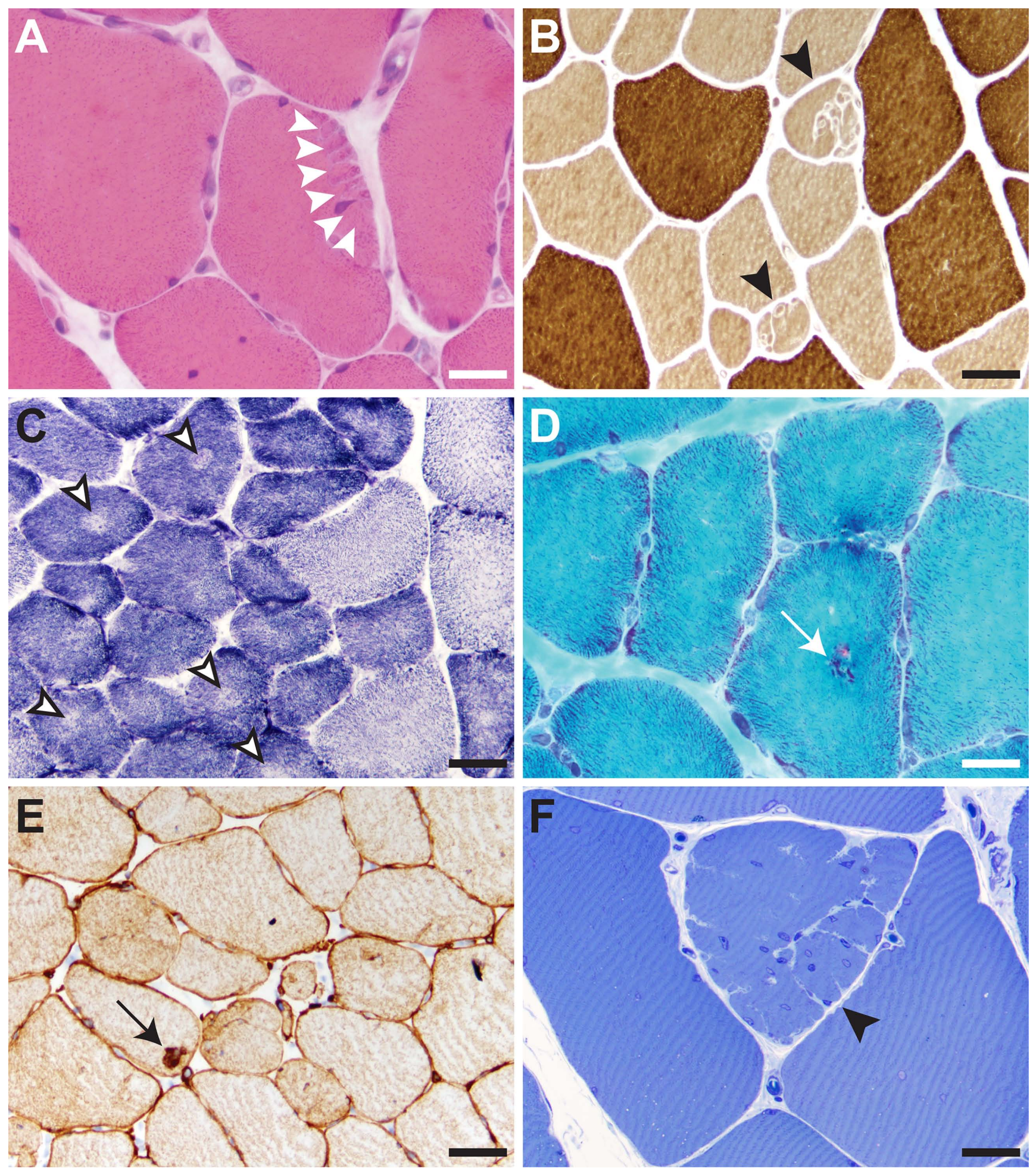
Figure 3

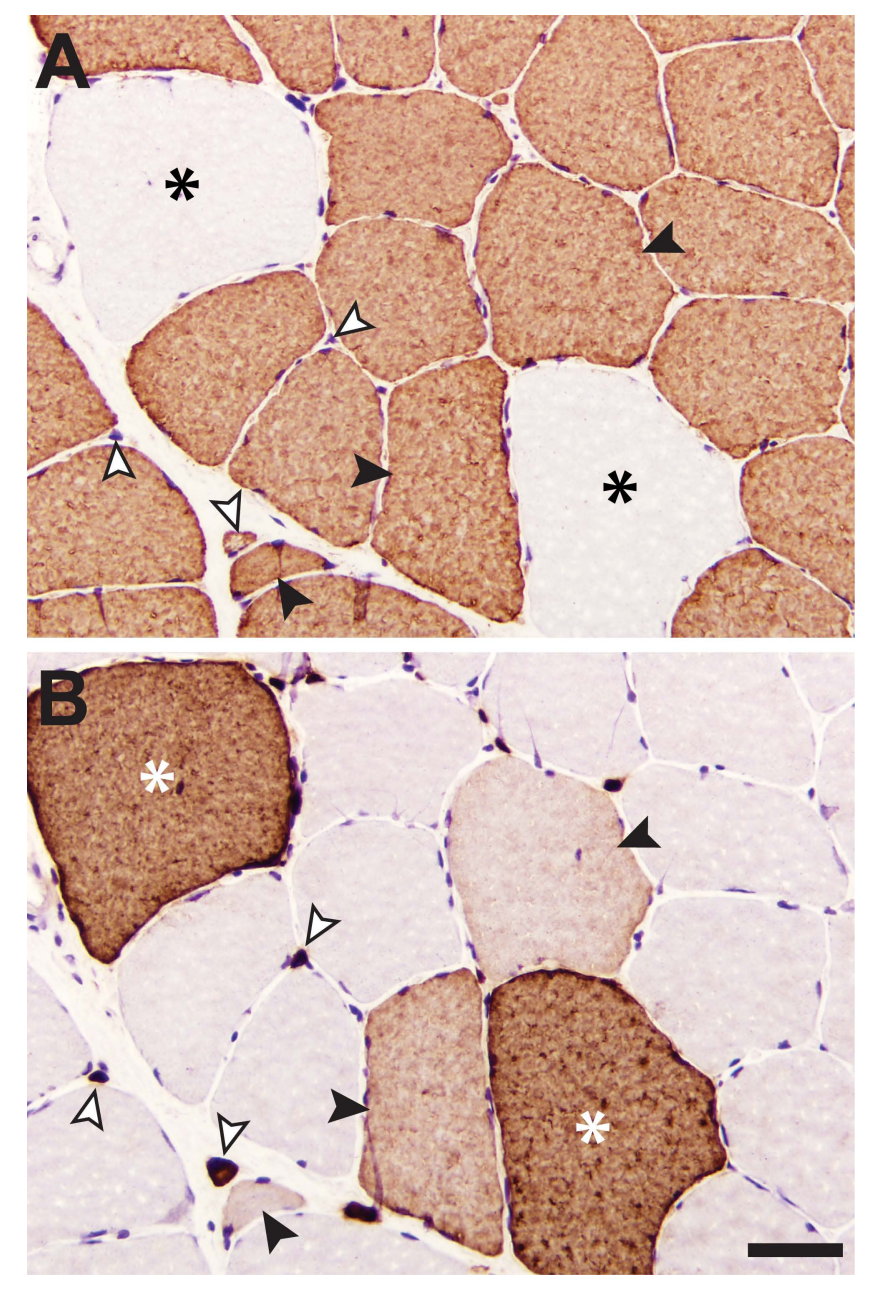


Figure 4
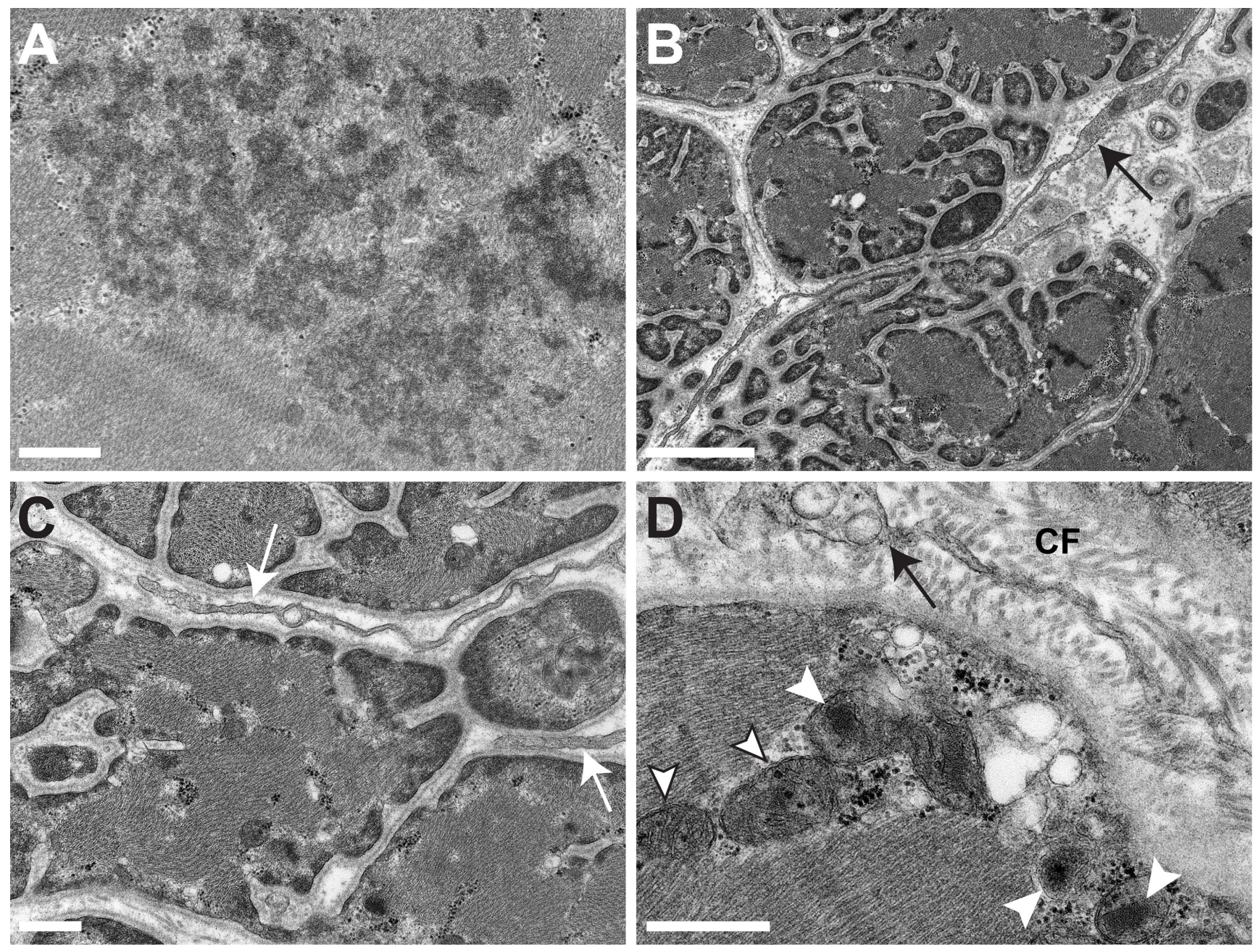
Figure 5

MYH7 exon 33 c.4522_4524delGAG (p.Glu1508del)

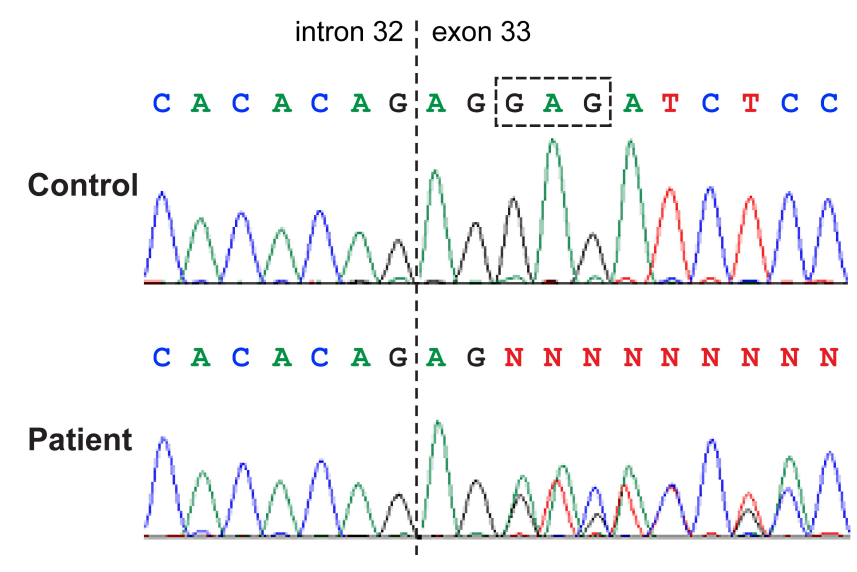

